\title{
Interpretation of UHECR mass composition based on CONEX simulations with revised inelastic cross sections
}

\section{Z. Plebaniak ${ }^{* a}$, T. Wibig ${ }^{b}$}

${ }^{a}$ National Centre for Nuclear Research, Astrophysics Division, Cosmic Ray Laboratory, ul. 28 Putku Strzelców Kaniowskich 69, 90-558 Łódź, Poland.

${ }^{b}$ Faculty of Physics and Applied Informatics, University of ódz, Poland

E-mail: zp@zpk.u.lodz.pl

\begin{abstract}
Cosmic rays with energies exceeding $10^{20} \mathrm{eV}$ have been detected thanks to indirect measurements of primary particles by EAS observations. Thanks to increasing number of events registered by fluorescence observatories like Telescope Array or Pierre Auger Observatory, we can try to solve the main problems in cosmic rays physics. One of them, understanding of the mass composition, require precise interpretation of measured EAS picture using monte-carlo simulations. Inelastic cross-section for proton-air and nucleus-air is one of parameters plays main role during EAS development and because of strong impact on $\mathrm{X}_{\max }$ position is very important for interpretation of mass composition of primary particles. In this work, we present results of mass composition analysis based on CONEX simulations with three commonly used high energy interaction models - QGSJETII-04, Sibyl12.3c and EPOS-LHC. For simulations we used revised by us inelastic cross-sections extrapolated up to ultra high energies using geometrical model. As a results we present that interpretation of mass composition depends not only on used high energy model or experimental data set, but also on small differences in inelastic cross sections.
\end{abstract}

36th International Cosmic Ray Conference -ICRC2019-

July 24th - August 1st, 2019

Madison, WI, U.S.A.

${ }^{*}$ Speaker. 


\section{Introduction}

The development of EAS is phenomenon so complicated that the interpretation of its results requires complex Monte Carlo simulations. Computer programs for these simulations should accommodate different models of cascade development, and in particular, different models of the first, most energetic interaction. Currently used models, based on accelerator data at energies many times lower than those observed in cosmic rays. It is required to extrapolate parameters of these models far to unmeasured energies. In this paper we present way for extrapolation of inelastic nucleus-nucleus cross-section using prepared by us model of elastic scattering in geometrical picture. Based on this model, nucleus-nucleus cross sections have been calculated and implemented in CONEX [1] program inside three quite popular high energy interaction models - EPOS-LHC [2], Sibyll 2.3c [3, 4] and QGSJETII-04 [5]. Using these models, EAS development simulations have been performed. Finally, we present discussion regarding interpretation of cosmic rays mass composition in case of original and recalculated by us inelastic cross-sections.

\section{Optical Model of scattering and extrapolated cross-sections}

According to description of the optical model presented in [6], in case of nucleon-nucleon scattering, differential elastic cross-section is related to the square of the scattering amplitude $|F(s, t)|^{2}$. This may be expressed by:

$$
F(s, t)=i \int_{0}^{\infty} J_{0}(b \sqrt{-t}) \Gamma(s, b) b d b=i \int_{0}^{\infty} J_{0}(b \sqrt{-t})\{1-\exp [-\Omega(s, b)]\} b d b .
$$

Transmission coefficient, so-called eikonal function $\Omega(s, b)$ ) is defined by the convolution of two colliding hadrons profiles $D(b)$ in plane perpendicular to the beam axis, with fixed impact parameter b:

$$
D(\mathbf{b})=\int_{-\infty}^{\infty} \rho(x, y, z) d z
$$

We proposed the following form of the hadron matter distribution in nucleon:

$$
\rho_{h}(\mathbf{r})=\frac{1}{8 \pi}\left(c_{1} m_{1}^{3} e^{-m_{1}|\mathbf{r}|}+c_{2} m_{2}^{3} e^{-m_{2}|\mathbf{r}|}\right) .
$$

As a result, we have four energy-depends parameters model of hadron matter distribution. $c_{1}$ and $c_{2}$ are normalization constants while $m_{1}$ and $m_{2}$ are slopes of two proposed exponential functions. Next step was to obtain correct values of these parameters. It have been done based on the differential elastic cross section distributions measured in accelerators in the energy range from $10 \mathrm{GeV}$ to $8 \mathrm{TeV}$ in c.m.s. In the Fig. 1 we present revised values of these parameters. Accuracy of these fits are presented for three selected energies in Fig. 2 (left) by comparison of calculated amplitudes with experimental data. 

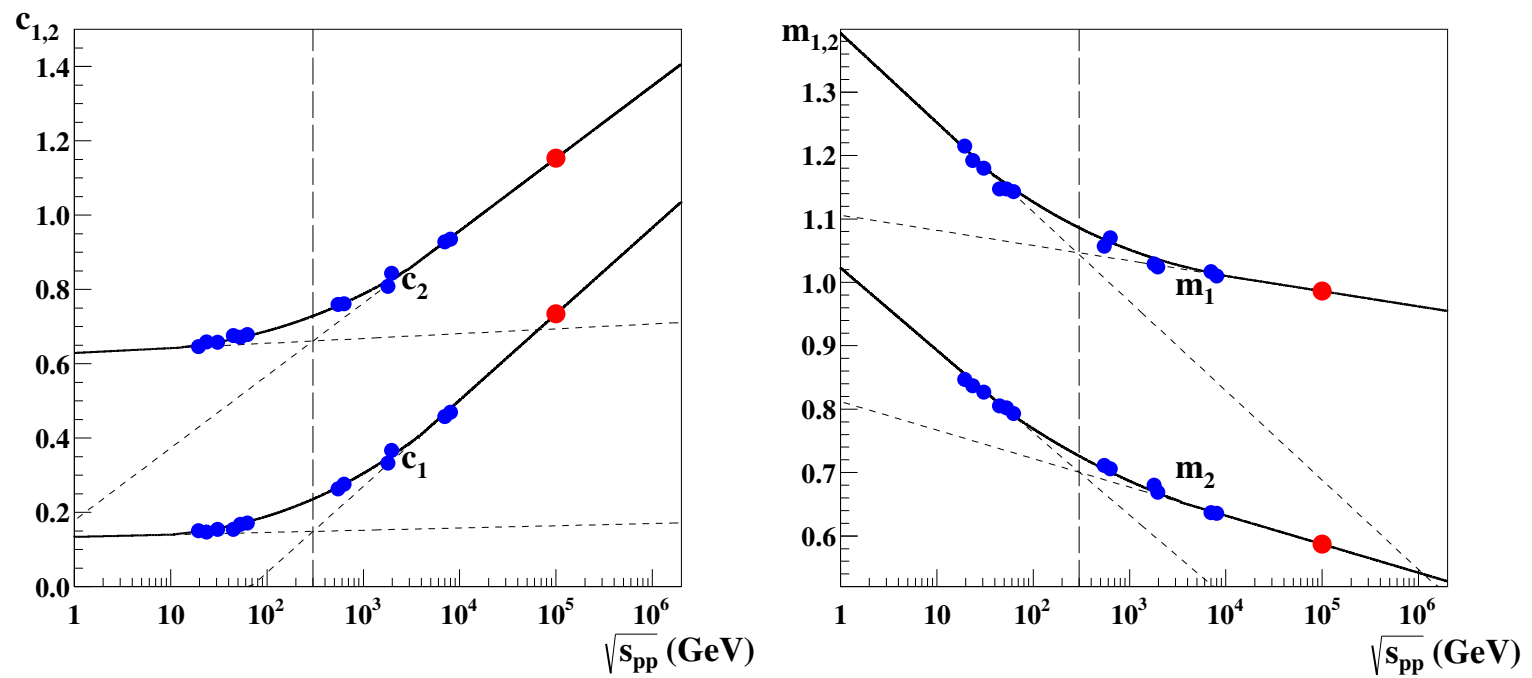

Figure 1: Four energy depends parameters of hadron matter distribution $\rho_{h}(\mathbf{r}) . c_{1,2}$ represents normalization constants while $m_{1,2}$ represents slopes of the exponential fractions.

In general, eikoanl is a complex function. Relation between real and imaginary part is introduced by energy-depends function $\lambda(s)$, representing real part of eikonal:

$$
\chi(b, s)=(i+\lambda(s)) \cdot \Omega(b)
$$
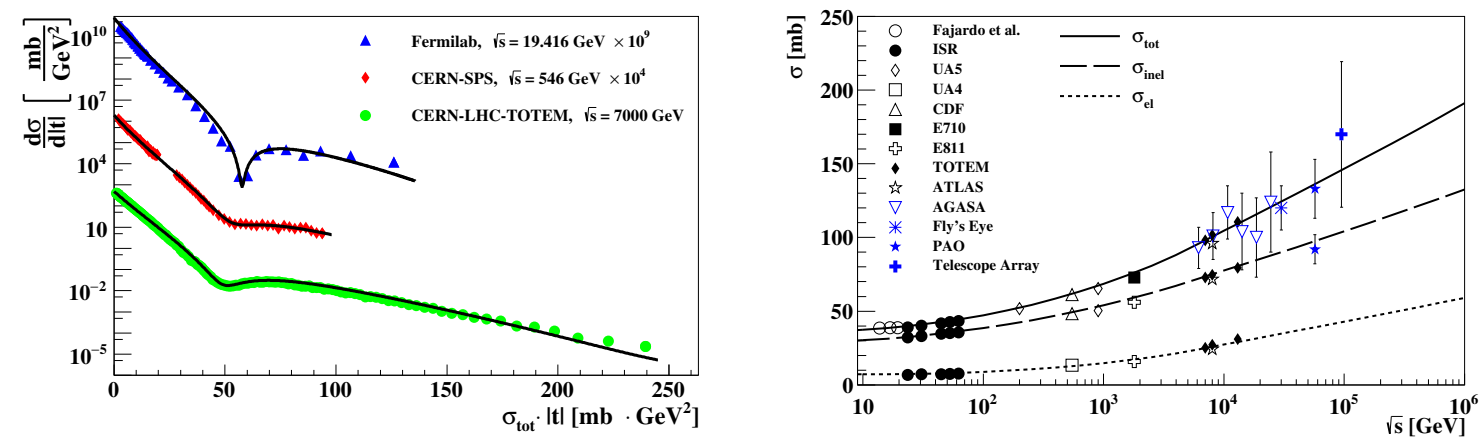

Figure 2: Proton-proton amplitudes calculated with model and compared with experimental data for three selected energies (left), and calculated elastic, inelastic and total cross-sections compared with experimental data in wide energy range (right)

Finally, with $\chi(b, s)$ we can express elastic, inelastic and total cross-section for proton-proton scattering by:

$$
\begin{gathered}
\sigma_{t o t}(s)=2 \int\left[1-\Re\left(e^{i \chi(b, s)}\right)\right] d^{2} \mathbf{b} \\
\sigma_{e l}(s)=\int\left[1-e^{i \chi(b, s)}\right]^{2} d^{2} \mathbf{b}
\end{gathered}
$$




$$
\sigma_{\text {inel }}(s)=\int 1-\left[e^{i \chi(b, s)}\right]^{2} d^{2} \mathbf{b}
$$

Taking parameters plotted in Fig. 1 and using equations 2.5, 2.6 and 2.7, the discussed crosssections for proton-proton collisions have been calculated. Results are presented and compared with experimental data in Fig. 2 (right)

In the last step, we have calculated inelastic cross-sections for the nucleus-Air case, based on the pure Glauber theory. Taking into account, previously presented hadron matter distribution, we used Woods-Saxon distribution of the nucleons in the nuclei. As a result in Fig. 3 we present calculated cross-sections in case of proton-Air (left), and Fe-Air (right) interactions. Air is a composition of $\mathrm{He}, \mathrm{O}$, and $\mathrm{N}$ nuclei with appropriate weight. Results are presented in comparison with related values of cross-sections used in QGSJETII-04, EPOS-LHC and Sibyll2.3c high energy interaction models.
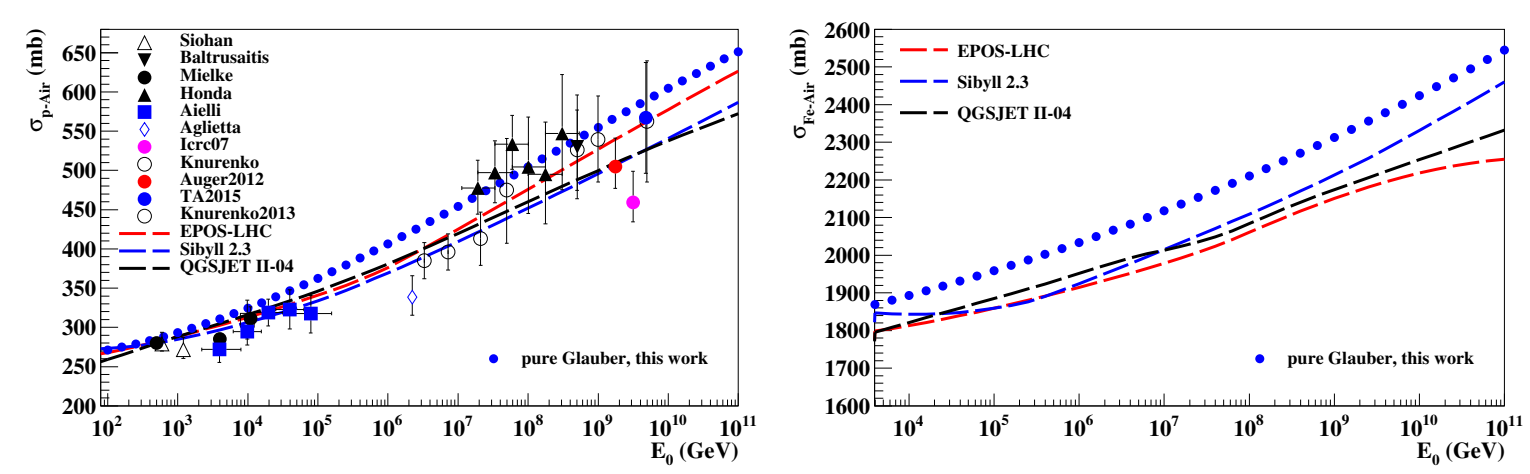

Figure 3: Calculated inelastic cross-sections in case of p-Air (left) and Fe-Air (right) interactions, compared with valued used in three models in CONEX software.

\section{Simulations of EAS development with CONEX}

Calculated cross-sections have been implemented to the three high energy interaction models - QGSJETII-04, EPOS-LHC and Sibyll 2.3c to perform simulations of the longitudinal EAS development. Models have been not modified. The only modification concerned $\sigma_{p-A i r}^{\text {inel }}$ and $\sigma_{N-A i r}^{\text {inel }}$ cross-sections. Then, we performed simulations in four points on the energy scale from the $10^{8}$ $\mathrm{GeV}$ to the $10^{11} \mathrm{GeV}$ in the laboratory system. For each energy, we simulated 500 showers.

\section{Mass Composition}

To determine mass composition based on performed simulations we chose following solution: First, obtained $\mathrm{X}_{\max }$ distributions have been fitted by the two free parameters function in the form:

$$
F_{\Gamma}\left(X_{\max }\right)=\frac{\left(X_{\max }-p\right)^{k-1}}{\Gamma(k) \Theta^{k}} \cdot \exp \left(-\frac{\left(X_{\max }-p\right)}{\Theta}\right)
$$

with $\mathrm{k}=5$.

This operation repeated for each data set gives us values of $\Theta$ and $p$ parameters as a function of the 

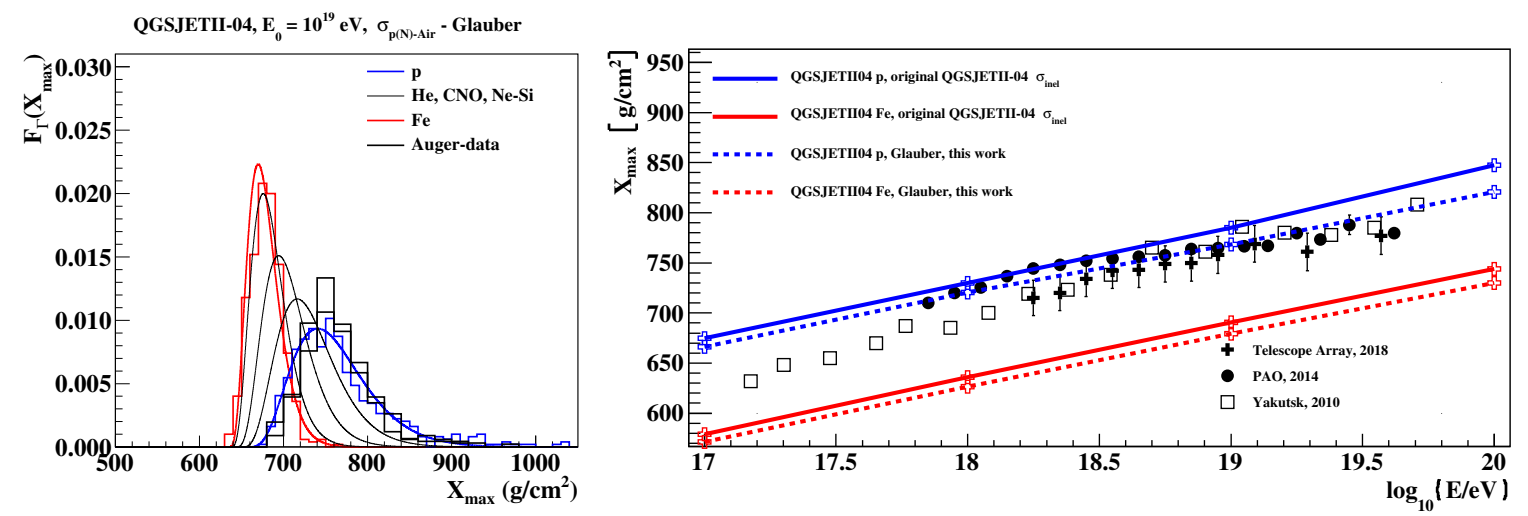

Figure 4: Example of obtained $\mathrm{X}_{\max }$ values for QGSJETII-04 model. On the left fitted by $F_{\Gamma}\left(X_{\max }\right)$ function distributions of $\mathrm{X}_{\max }$ in case of Glauber approach at $\mathrm{E}_{0}=10^{19} \mathrm{eV}$. On the right - average values of $\mathrm{X}_{\max }$ from simulations in case of proton (blue lines) and iron (red lines) as a primary particles.

atomic weight for all energies. Thin black lines in the Fig. 4 (left) represents the $F_{\Gamma}\left(X_{\max }\right)$ function obtained by the interpolation of their parameters for three groups of nuclei: $\mathrm{He}, \mathrm{CNO}$ and $\mathrm{Ne}-\mathrm{Si}$. Composition of these functions separately for each energy, interaction models and cross-section model (original or our Glauber) were used for fitting of measured $\mathrm{X}_{\max }$ distributions published by Telescope Array [7] and PAO [8] experiments for several points on the energy scale. As a final result, we obtained the preliminary values of the average mass number for each energy measured by the experiments. Results of primary particles mass composition for three analyzed models in case of Telescope Array and PAO experiment are presented in Figs. 5, 6 and 7.
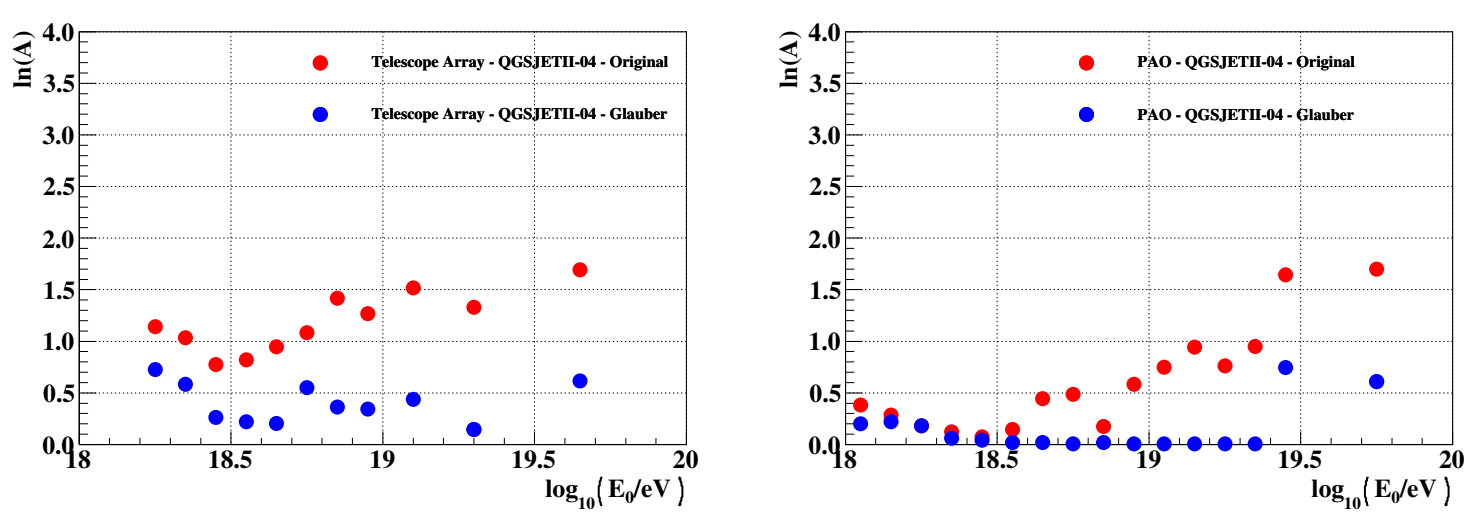

Figure 5: Mass composition for QGSJETII-04 model in case of Telescope Array data (left) and Pierre Auger Observatory (right). Different colors represents two models of inelastic cross section extrapolations. 

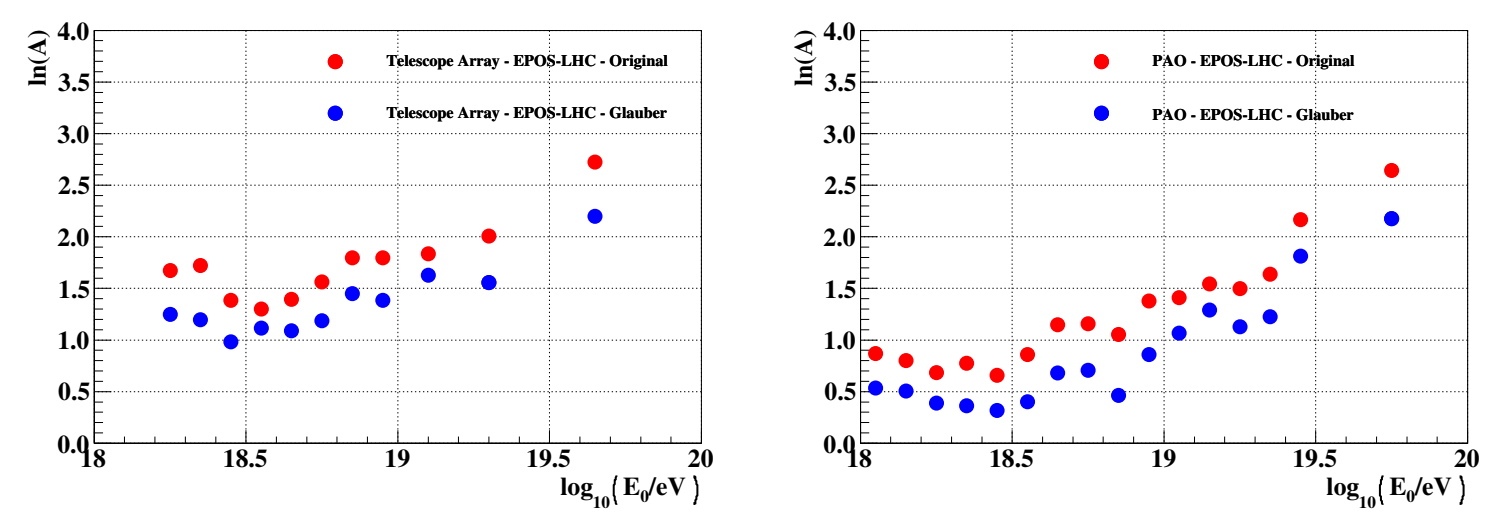

Figure 6: Mass composition for EPOS-LHC model in case of Telescope Array data (left) and Pierre Auger Observatory (right). Different colors represents two models of inelastic cross section extrapolations.
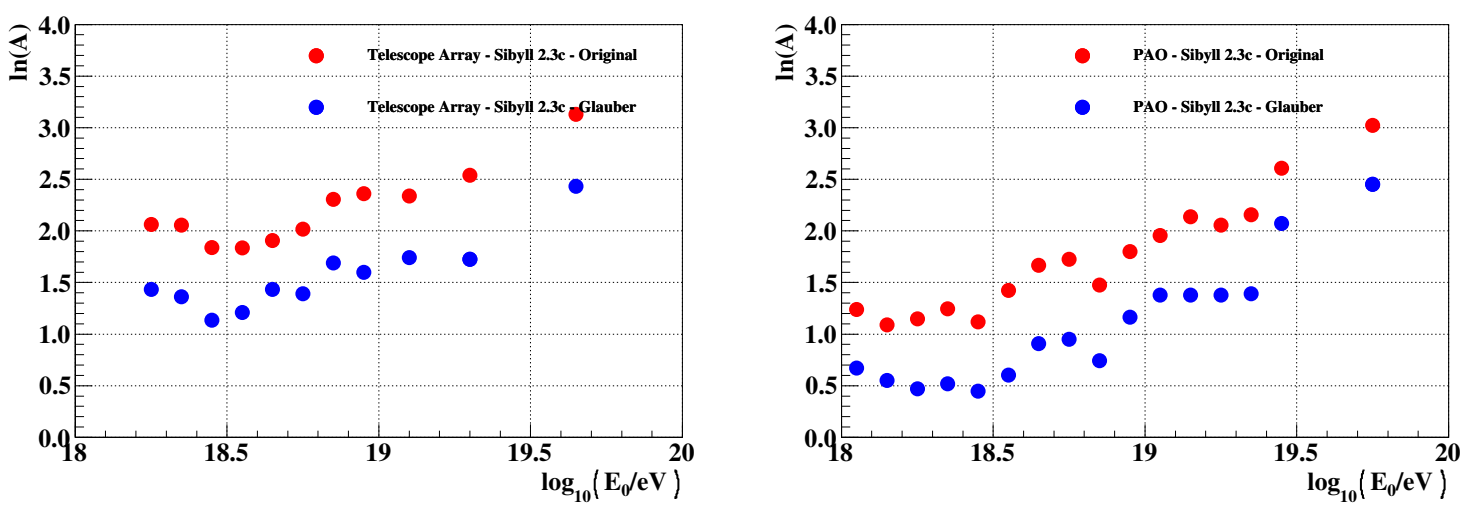

Figure 7: Mass composition for Sibyll 2.3c model in case of Telescope Array data (left) and Pierre Auger Observatory (right). Different colors represents two models of inelastic cross section extrapolations.

\section{Conclusions}

In this work we presented revised model of the extrapolation of inelastic cross sections for nucleus-nucleus interactions up to cosmic ray energies. Calculated cross sections indicates good agreement with experimental data in case of proton-proton scattering, what assuming the correctness of the Glauber approach let us believe that nucleus-nucleus cross-sections are also calculated in the proper way. Calculated cross-section have been implemented in simulation program - CONEX, and in case of three commonly used high energy interaction models, simulations of longitudinal EAS development have been performed. One conclusion is the most important: interpretation of the primary cosmic rays mass composition from measured EAS signal strongly depends on the high energy interaction model and experiment. Correct description and extrapolation of the high energy models parameters like inelastic cross-section plays very important role in the interpretation of the taken data.

Acknowledgments: This work was supported by grant no 2015/19/N/ST9/03708 funded by National Science Centre in Poland. 


\section{References}

[1] T. Pierog, M.K. Alekseeva, T. Bergmann, V. Chernatkin, R. Engel, D. Heck, N.N. Kalmykov, J. Moyon, S. Ostapchenko, T. Thouw, and K. Werner. First results of fast one-dimensional hybrid simulation of eas using conex. Nuclear Physics B - Proceedings Supplements, 151(1):159 - 162, 2006. VERY HIGH ENERGY COSMIC RAY INTERACTIONS.

[2] T. Pierog, Iu. Karpenko, J. M. Katzy, E. Yatsenko, and K. Werner. Epos lhc: Test of collective hadronization with data measured at the cern large hadron collider. Phys. Rev. C, 92:034906, Sep 2015.

[3] Felix Riehn, Hans P. Dembinski, Ralph Engel, Anatoli Fedynitch, Thomas K. Gaisser, and Todor Stanev. The hadronic interaction model SIBYLL 2.3c and Feynman scaling. PoS, ICRC2017:301, 2018.

[4] Eun-Joo Ahn, Ralph Engel, Thomas K. Gaisser, Paolo Lipari, and Todor Stanev. Cosmic ray interaction event generator sibyll 2.1. Phys. Rev. D, 80:094003, Nov 2009.

[5] S. Ostapchenko. Monte carlo treatment of hadronic interactions in enhanced pomeron scheme: Qgsjet-ii model. Phys. Rev. D, 83:014018, Jan 2011.

[6] Zbigniew Plebaniak and Tadeusz Wibig. Elastic scattering in geometrical model. Physics Letters B, 761:469-474, 2016.

[7] R. U. Abbasi et al. Depth of Ultra High Energy Cosmic Ray Induced Air Shower Maxima Measured by the Telescope Array Black Rock and Long Ridge FADC Fluorescence Detectors and Surface Array in Hybrid Mode. Astrophys. J., 858(2):76, 2018.

[8] Alexander Aab et al. Depth of maximum of air-shower profiles at the Pierre Auger Observatory. I. Measurements at energies above 10 $0^{17.8}$ eV. Phys. Rev., D90(12):122005, 2014. 